\title{
Integer Programming Duality in Multiple Objective Programming
}

\author{
Kathrin Klamroth $^{1} \quad$ Jørgen Tind ${ }^{1} \quad$ Sibylle Zust $^{2}$
}

03.07 .2003

\begin{abstract}
The weighted sums approach for linear and convex multiple criteria optimization is well studied. The weights determine a linear function of the criteria approximating a decision makers overall utility. Any efficient solution may be found in this way. This is not the case for multiple criteria integer programming. However, in this case one may apply the more general $e$-constraint approach, resulting in particular single-criteria integer programming problems to generate efficient solutions. We show how this approach implies a more general, composite utility function of the criteria yielding a unified treatment of multiple criteria optimization with and without integrality constraints. Moreover, any efficient solution can be found using appropriate composite functions. The functions may be generated by the classical solution methods such as cutting plane and branch and bound algorithms.
\end{abstract}

Keywords: multiple objective integer programming, duality, utility functions

\section{Introduction}

Multiple objective linear- and integer programming problems play a central role in the field of multicriteria optimization. Besides their relevance for practical applications, they are theoretically interesting due to their intrinsic relationship to the classical theory of (single objective) linear and integer programming. Integer programming problems with multiple objectives are particularly challenging since, due to their nonconvexity, weighted sums scalarizations of the objective

\footnotetext{
${ }^{1}$ Department of Statistics and Operations Research, Institute for Mathematical Sciences, University of Copenhagen, Denmark.

${ }^{2}$ Los Alamos National Laboratory, Mailstop M997, Los Alamos, NM 87545, USA.
} 
functions cannot be used to generate all nondominated solutions. Besides supported, there exist nonsupported nondominated solutions that are dominated by fractional convex combinations of other solutions. Since nonsupported nondominated solutions often make up the majority of nondominated solutions of discrete problems, they cannot be ignored in the decision process (see Visée et al., 1998, for example data on bicriteria knapsack problems).

A multitude of different techniques for the generation of all the nondominated solutions of multiple objective integer programming problems (MOIPs) have been suggested in the recent literature. For an overview we refer to Clímaco et al. (1997). Particularly relevant in the context of this paper are the $e$-constraint method introduced in Haimes et al. (1971) (see also Cohon, 1978; Chankong and Haimes, 1983a,b, for further discussions), duality results for single objective linear and integer programming problems (see, for example, Schrijver, 1986; Wolsey, 1998) and cutting plane and branch and bound algorithms (see, for example, Nemhauser and Wolsey, 1986). An application of the $e$-constraint method in the context of (MOIP) was mentioned in Bard (1986), and cutting plane approaches and branch and bound algorithms were applied to (MOIP) by Alves and Clímaco (1999) and Alves and Clímaco (2000). The latter describe the combination of an interactive method based on a parameterization of the reference point with the repeated solution of non-weighted Tchebycheff programs, using a cutting planes approach and a branch and bound methodology, respectively. From the perspective of single objective linear and integer programming, duality theory has a long tradition and a large variety of applications. Among others, Wolsey (1981), Llewellyn and Ryan (1993) and Klabjan (2002) discussed generation methods for optimal dual functions for single objective integer programming problems. An introduction to duality theory in the context of convex multiple objective programming is, for example, given in Jahn (1983).

The goal of this paper is to use dual information from single objective linear and integer programming for the development of an integrated utility theory that applies not only to multiple objective linear programming (MOLP) but also to multiple objective integer programming (MOIP). A natural generalization of the weighted sums approach for (MOLP) to a composite utility function approach for (MOIP) is developed that allows a unified treatment of multiple objective linear and integer programming problems. This combination of ideas from integer programming duality with methods from multicriteria programming and utility theory is new and it implies, among others, meaningful bounds on the complete efficient set of (MOIPs) in the objective space.

After a formal introduction of the problem in Section 2, interrelations between linear programming duality and the weighted sums approach for (MOLP) are reviewed in Section 3. In Section 4, integer programming duality is used to derive a multiobjective integer programming analogue to the weighted sums approach. In particular, we analyze how the weighting vectors - containing the utility information in the case of (MOLP) - can be deduced from optimal dual variables of 
appropriately formulated e-constraint problems. Similarly, composite functions representing the utility information in the integer case can be deduced from optimal dual functions of correspondingly defined $e$-constraint problems for (MOIP). Besides the fact that utility information becomes explicitly available with these composite functions, it is also shown that level sets of composite functions can be used to obtain global bounds on the efficient set of (MOIP). The application of cutting plane methods and branch and bound algorithms for the practical determination of composite functions for (MOIP) is discussed in Section 5. The paper is concluded in Section 6 with an outlook on potential incorporations and applications of composite functions within the framework of interactive decision support algorithms for (MOIP).

\section{Problem Formulation}

The following notation is used throughout the paper. Let $u, w \in \mathbb{R}^{k}$ be two vectors. We denote components of vectors by subscripts and enumerate vectors by superscripts. $u>w$ denotes $u_{i}>w_{i}$ for all $i=1, \ldots, k . u \geq w$ denotes $u_{i} \geq w_{i}$ for all $i=1, \ldots, k$, but $u \neq w$. $u \geqq w$ allows equality. The symbols $<, \leq, \leqq$ are used accordingly. Let $\mathbb{R}_{\leqq}^{k}:=\left\{x \in \mathbb{R}^{k}: x \leqq 0\right\}$. The set $\mathbb{R}_{\geqq}^{k}$ is defined accordingly and the set $u+\mathbb{R}_{\geq}^{k}$, where $u \in \mathbb{R}^{k}$, is referred to as a dominating cone. For an $m \times n$ - matrix $A$, let $\left(a_{i \bullet}\right)=: a^{i}$ be the $i$ th row of $A$, and let $\left(a_{\bullet j}\right)$ be the $j$ th column of $A$. Moreover, for an index set $J \subset\{1, \ldots, m\}, A_{J}$ denotes the submatrix $\left(a^{j}\right)_{j \in J}$ of $A$, i.e., the submatrix consisting of the rows in $J$.

We consider the following general multiple objective program (MOP)

$$
\begin{array}{ll}
\max & \left\{z_{1}=f_{1}(x)\right\} \\
& \vdots \\
\max & \left\{z_{k}=f_{k}(x)\right\} \\
\text { s.t. } & x \in S,
\end{array}
$$

where $S \subseteq \mathbb{R}^{n}$ is the feasible set and $f_{i}(x), i=1, \ldots, k$, are real-valued functions. Problem (1) is called a multiple objective linear program (MOLP) if

$$
\begin{aligned}
& f_{i}(x)=c^{i} x \quad \forall i=1, \ldots, k \quad \text { and } \\
& S=\left\{x \in \mathbb{R}^{n}: A x \leqq b, x \geqq 0\right\}
\end{aligned}
$$

where we assume that all components of the $k \times n$ - matrix $C:=\left(c^{i}\right)_{i=1}^{k}$, the $m \times n$ - matrix $A$ and the $m$ - vector $b$ are integer. Thus, the MOLP can be written as

$$
\begin{array}{ll}
\operatorname{vmax} & C x \\
\text { s.t. } & A x \leqq b \\
& x \geqq 0 .
\end{array}
$$


Similarly, problem (1) is called a multiple objective integer program (MOIP) if

$$
\begin{aligned}
& f_{i}(x)=c^{i} x \quad \forall i=1, \ldots, k \text { and } \\
& S=\left\{x \in \mathbb{R}^{n}: A x \leqq b, x \geqq 0 \text { and integer }\right\} .
\end{aligned}
$$

Problem (1) then reads

$$
\begin{array}{ll}
\operatorname{vmax} & C x \\
\text { s.t. } & A x \leqq b \\
& x \geqq 0 \text { and integer. }
\end{array}
$$

We define the set of all feasible criterion vectors $Z$, the set of all (globally) nondominated criterion vectors $N$ and the set of all efficient points $E$ of (1) as follows

$$
\begin{aligned}
& Z=\left\{z \in \mathbb{R}^{k}: z=f(x), x \in S\right\}=f(S) \\
& N=\{z \in Z: \nexists \tilde{z} \in Z \text { s.t. } \tilde{z} \geq z\} \\
& E=\{x \in S: f(x) \in N\},
\end{aligned}
$$

where $f(x)=\left(f_{1}(x), \ldots, f_{k}(x)\right)^{T}$. We assume that the set $Z$ is bounded and $\mathbb{R}_{\leqq}^{k}$ closed, i.e., the set $Z+\mathbb{R}_{\leq}^{k}$ is closed, and that the sets $N$ and $E$ are nonempty. A point $\bar{x} \in S$ is called wea $\overline{\bar{k}} l y$ efficient if there does not exist another point $\hat{x} \in S$ such that $f(\hat{x})>f(\bar{x})$.

\section{Methodology}

\subsection{Solution Approaches for MOPs and MOLPs}

The following results can, for example, be found in Steuer (1986).

\subsubsection{Weighted-Sums Approach}

Let $\Lambda:=\left\{\lambda \in \mathbb{R}^{k}: \lambda>0, \sum_{i=1}^{k} \lambda_{i}=1\right\}$ be the set of all strictly positive weighting vectors. Then for a fixed $\bar{\lambda} \in \Lambda$ the composite or weighted-sums program corresponding to (1) is given by

$$
\begin{array}{ll}
\max & \bar{\lambda}^{T} f(x) \\
\text { s.t. } & x \in S .
\end{array}
$$

Theorem 1. If $x^{*}$ is an optimal solution of (6), then $x^{*}$ is efficient for (1).

Theorem 2. If $x^{*}$ is an efficient solution for the MOLP (3), then there exists $\bar{\lambda} \in \Lambda$ such that $x^{*}$ is optimal for (6).

Note that Theorem 2 does not generalize to nonlinear or discrete MOPs.

Corollary 1. If $\bar{\lambda} \in \bar{\Lambda}:=\left\{\lambda \in \mathbb{R}^{k}: \lambda \geq 0, \sum_{i=1}^{k} \lambda_{i}=1\right\}$, then an optimal solution $x^{*}$ of (6) is weakly efficient for (1). 


\subsection{2 e-Constraint Approach}

Let $i \in\{1, \ldots, k\}$ and $e_{j} \in \mathbb{R}, j \in J_{i}:=\{1, \ldots, k\} \backslash\{i\}$. Then the $i$ th objective e-constraint program can be formulated as

$$
\begin{array}{ll}
\max & f_{i}(x) \\
\text { s.t. } & f_{j}(x) \geq e_{j} \quad \forall j \in J_{i} \\
& x \in S .
\end{array}
$$

We will assume in the following that the lower bounds $e_{j}, j \in J_{i}$ are always chosen such that (7) is feasible. This implies the existence of an optimal solution as $Z$ is assumed to be bounded.

Theorem 3. Every optimal solution of (7) is a weakly efficient solution of (1), and the set of all optimal solutions of (7) contains at least one efficient solution of (1).

Theorem 4. If $x^{*} \in S$ is an efficient solution of (1), then there exists an index $i \in\{1, \ldots, k\}$ and lower bounds $e_{j} \in \mathbb{R}, j \in J_{i}$ such that $x^{*}$ is an optimal solution of (7).

Observe that Theorem 4 holds for general MOPs. It follows that:

Corollary 2. If there exists an index $i$ and lower bounds $e_{j} \in \mathbb{R}, j \in J_{i}$ such that $x^{*}$ is the unique optimal solution of (7), then $x^{*}$ is efficient for (1).

\subsection{Linear Programming Duality and its Implications for MOLPs}

For MOLPs we can find a close relationship between the weighted sums approach and the $e$-constraint approach that is based on linear programming duality.

Consider the $e$-constraint LP

$$
\begin{array}{ll}
\max & c^{i} x \\
\text { s.t. } & c^{j} x \geq e_{j} \quad \forall j \in J_{i} \\
& A x \leqq b \\
& x \geqq 0 .
\end{array}
$$

Then we can introduce dual variables $u_{j}, j \in J_{i}$ and $y \in \mathbb{R}^{m}$ to formulate the dual of (8) as

$$
\begin{array}{ll}
\min & -\sum_{j \in J_{i}} e_{j} u_{j}+b^{T} y \\
\text { s.t. } & -\sum_{j \in J_{i}} c_{l}^{j} u_{j}+\left(A^{T} y\right)_{l} \geq c_{l}^{i} \quad \forall l=1, \ldots, n \\
& u_{j} \geq 0 \forall j \in J_{i}, \quad y \geqq 0 .
\end{array}
$$


Using only those optimal dual variables $u_{j}^{*}, j \in J_{i}$ of $(9)$, that correspond to the $e$-constraints of (8), we can define a weighting vector $\hat{u} \in \mathbb{R}^{k}$ as

$$
\hat{u}_{j}:= \begin{cases}u_{j}^{*}, & j \in J_{i} \\ 1, & j=i\end{cases}
$$

After normalization we obtain $\bar{\lambda}:=\frac{\hat{u}}{\|\hat{u}\|}$ with $\bar{\lambda} \in \bar{\Lambda}$. It will be shown below that an optimal solution of (8) is also optimal for (6) which in the case of MOLPs is given by

$$
\begin{array}{ll}
\max & \bar{\lambda}^{T} C x \\
\text { s.t. } & A x \leqq b \\
& x \geqq 0
\end{array}
$$

or, equivalently,

$$
\begin{array}{ll}
\max & \hat{u}^{T} C x \\
\text { s.t. } & A x \leqq b \\
& x \geqq 0 .
\end{array}
$$

Theorem 5. Let $x^{*} \in S$ be an optimal solution of (8). Then the dual optimal solution can be used to construct a weighting vector $\bar{\lambda} \in \bar{\Lambda}$ such that $x^{*}$ is also optimal for (11).

Proof. To compare with the later developments we give a complete proof here even though the result is well known in the context of partial Lagrangian relaxation.

Let $x^{*}$ and $u^{*}, y^{*}$ be primal and dual optimal for (8) and (9), respectively. Hence $c^{j} x^{*} \geq e_{j} \forall j \in J_{i}$ and $-\sum_{j \in J_{i}} c_{l}^{j} u_{j}^{*}+\left(A^{T} y^{*}\right)_{l} \geq c_{l}^{i} \forall l=1, \ldots, n$ and, by linear programming duality, we have that $-\sum_{j \in J_{i}} e_{j} u_{j}^{*}+b^{T} y^{*}=c^{i} x^{*}$. Define $\hat{u}$ according to (10). It follows that

$$
b^{T} y^{*}=\sum_{j \in J_{i}} e_{j} u_{j}^{*}+c^{i} x^{*} \leq \sum_{j \in J_{i}}\left(c^{j} x^{*}\right) u_{j}^{*}+c^{i} x^{*}=\hat{u}^{T} C x^{*} .
$$

Now consider the dual of (12), i.e.,

$$
\begin{array}{ll}
\min & b^{T} y \\
\text { s.t. } & A^{T} y \geqq C^{T} \hat{u} \\
& y \geqq 0 .
\end{array}
$$

Since $y^{*}$ is dual feasible, and using again linear programming duality, we have

$$
b^{T} y^{*} \geq \hat{u}^{T} C x^{*}
$$

We can conclude that $b^{T} y^{*}=\hat{u}^{T} C x^{*}$, hence proving the optimality of $x^{*}$ for (12).

Conversely, the following result can be proven: 
Theorem 6. Let $x^{*} \in S$ be an optimal solution of (11) for some $\bar{\lambda} \in \bar{\Lambda}$. Then there exists an index $i \in\{1, \ldots, k\}$ such that $x^{*}$ is also optimal for (8), where the lower bounds are defined as $e_{j}:=c^{j} x^{*}, j \in J_{i}$.

Proof. Similar to the proof of Theorem 5 the following proof is included for completeness.

Let $x^{*}$ be optimal for (11). Hence $x^{*}$ is weakly efficient for (3) by Corollary 1. If $x^{*}$ is efficient, an arbitrary objective $i \in\{1, \ldots, k\}$ can be selected for (8). Otherwise, we select an index $i \in\{1, \ldots, k\}$ such that there exists no $\hat{x} \in S$ with $c^{i} \hat{x}>c^{i} x^{*}$ and $C_{J_{i}} \hat{x} \geqq C_{J_{i}} x^{*}$. In both cases it can be shown that $x^{*}$ is optimal for (8).

We will use later the fact that the maximization of the weighting function $\bar{\lambda}^{T} C x$ in (12) is equivalent to the maximization of the linear utility function

$$
g(x):=c^{i} x+\sum_{j \in J_{i}} u_{j}^{*} c^{j} x-b^{T} y^{*}=: c^{i} x-F^{*}\left(-C_{J_{i}} x, b\right),
$$

where $F^{*}: \mathbb{R}^{k-1+m} \rightarrow \mathbb{R}$ is a linear function (and thus nondecreasing and superadditive, c.f. Section 3.3), and $C_{J_{i}}$ denotes the submatrix of $C$ consisting of the rows in $J_{i}$, i.e., obtained by deleting its $i$ th row. Note that $b^{T} y^{*}$ is a constant that has no impact on the maximization of $g$.

\subsection{Integer Programming Duality}

The previous section shows that duality is a powerful tool for the generation of weighting vectors and hence of utility functions for MOPs. Since our ultimate goal is to develop a similar theory also for MOIPs, we will review some of the central concepts of integer programming duality in this section. A more detailed discussion of the subject, including proofs for the theorems stated below, can be found in Wolsey (1981) and Tind and Wolsey (1981).

Let $\mathcal{F}$ be the set of all nondecreasing functions $F: \mathbb{R}^{m} \rightarrow \mathbb{R}$, i.e.

$$
\mathcal{F}:=\left\{\left(F: \mathbb{R}^{m} \rightarrow \mathbb{R}\right): F(a) \leq F(b) \forall a, b \in \mathbb{R}^{m}, a \leqq b\right\}
$$

and consider the single objective integer programming problem

$$
\begin{array}{ll}
\max & c^{T} x \\
\text { s.t. } & A x \leqq b \\
& x \geqq 0 \text { and integer }
\end{array}
$$

with $c \in \mathbb{Z}^{n}$. Then it's dual can be written as

$$
\begin{array}{ll}
\min & F(b) \\
\text { s.t. } & F(A x) \geq c^{T} x \quad \forall x \geqq 0 \text { and integer } \\
& F \in \mathcal{F} .
\end{array}
$$


If the feasible set $\mathcal{F}$ is further restricted to the set $\mathcal{G}$ of nondecreasing and superadditive functions, i.e., to the set

$$
\begin{aligned}
\mathcal{G}:=\left\{\left(F: \mathbb{R}^{m} \rightarrow \mathbb{R}\right):\right. & F(0)=0,\left(F(a) \leq F(b) \forall a, b \in \mathbb{R}^{m}, a \leqq b\right) \\
& \text { and } \left.\left(F(a)+F(b) \leq F(a+b) \forall a, b \in \mathbb{R}^{m}\right)\right\}
\end{aligned}
$$

we can reformulate the dual of (14) as

$$
\begin{array}{ll}
\min & F(b) \\
\text { s.t. } & F\left(\left(a_{\bullet j}\right)\right) \geq c_{j} \quad \forall j=1, \ldots, n \\
& F \in \mathcal{G},
\end{array}
$$

where $\left(a_{\bullet j}\right)$ denotes the $j$ th column of $A$. Note that for a nondecreasing, superadditive function $F \in \mathcal{G}$, the constraint $F\left(\left(a_{\bullet} j\right)\right) \geq c_{j} \forall j=1, \ldots, n$ is equivalent to $F(A x) \geq c^{T} x \forall x \geqq 0$ and integer.

Theorem 7 (Weak Duality). $c^{T} x \leq F(b)$ for all primal feasible solutions $x$ of (14), and all dual feasible functions $F$ of (15) (or (16), respectively).

Theorem 8 (Strong Duality). If either (14) or (15) ((16), respectively) has a finite optimal solution, then there exists an optimal feasible solution $x^{*}$ of (14) and a dual optimal function $F^{*}$ of (15) ((16), respectively) such that $c^{T} x^{*}=F^{*}(b)$.

Moreover, if (14) is infeasible, then (15) ((16), respectively) is either infeasible or unbounded, and if (15) ((16), respectively) is infeasible, then (14) is either infeasible or unbounded.

The dual integer program and, in particular, the dual optimal functions $F^{*}$, are, among others, used for sensitivity analysis.

Corollary 3. Let $x^{*}$ and $F^{*}$ be primal and dual optimal for (14) and (15) / (16), respectively. If the constraint vector $b$ in (14) is changed to $b^{\prime} \neq b$ (for example, due to changes in the input data of a given problem) the function $F^{*}$ remains dual feasible also for the modified problem and satisfies $z^{\prime} \leq F^{*}\left(b^{\prime}\right)$, where $z^{\prime}$ denotes the optimal objective value of the modified problem.

\section{A Composite Function Approach to Multiple Objective Integer Programming}

We will in the following mostly refer to the more general formulation (15) of dual integer programming problems, c.f. Section 3.3. However, the following results equivalently hold for (16) in the case that we use superadditive functions.

Consider again the MOIP

$$
\begin{array}{ll}
\operatorname{vmax} & C x \\
\text { s.t. } & A x \leqq b \\
& x \geqq 0 \text { and integer. }
\end{array}
$$


Applying the $e$-constraint approach for its solution implies that we have to repeatedly solve problem (7) for different choices of an individual objective function $f_{i}(x)=c^{i} x, i \in\{1, \ldots, k\}$ and lower bound vectors $e:=\left(e_{j}\right)_{j \in J_{i}} \in \mathbb{R}^{k-1}$ bounding the remaining objectives. Problem (7) is thus given by

$$
\begin{array}{ll}
\max & c^{i} x \\
\text { s.t. } & C_{J_{i}} x \geqq e \\
& A x \leqq b \\
& x \geqq 0 \text { and integer, }
\end{array}
$$

and its dual can be written as

$$
\begin{array}{ll}
\min & F(-e, b) \\
\text { s.t. } & F\left(-C_{J_{i}} x, A x\right) \geq c^{i} x \quad \forall x \geqq 0 \text { and integer } \\
& F \in \mathcal{F},
\end{array}
$$

c.f. (15). Let $F^{*}$ be optimal for (18), that is, $F^{*}$ is a dual optimal function for (17). Similar to the application of the dual optimal solutions of (9) for the generation of a weighting vector and hence a linear utility function (13) for the MOLP (3) in Section 3.2, we will now use the dual optimal functions of (18) to define a utility function for the MOIP (5) as a composite function given by

$$
g(x):=c^{i} x-F^{*}\left(-C_{J_{i}} x, b\right) .
$$

The composite integer programming problem is thus formulated as

$$
\begin{array}{ll}
\max & c^{i} x-F^{*}\left(-C_{J_{i}} x, b\right) \\
\text { s.t. } & A x \leqq b \\
& x \geqq 0 \text { and integer. }
\end{array}
$$

Theorem 9. Let $x^{*}$ be optimal for (17) and let $F^{*}$ be optimal for (18). Then $x^{*}$ is also optimal for (20).

Proof. Let $F$ be feasible for (18). By Theorem 7 we have that $c^{i} x \leq F(-e, b)$ for all $x$ feasible for (17). Moreover, any $x$ that is feasible for (20) is also feasible for (17) if $e:=C_{J_{i}} x$, implying that $c^{i} x \leq F\left(-C_{J_{i}} x, b\right)$ for all $x$ feasible for (20). Thus the optimal objective value $z^{*}$ of $(20)$ satisfies $z^{*} \leq 0$.

Since $x^{*}$ is feasible for (17), it is also feasible for (20), and by Theorem 8 it satisfies $c^{i} x^{*}=F^{*}\left(-C_{J_{i}} x^{*}, b\right)$. This proves the optimality of $x^{*}$ for $(20)$.

Theorem 10. $x^{*}$ is an efficient solution of (5) if and only if there exists an index $i \in\{1, \ldots, k\}$ and a nondecreasing function $F^{*} \in \mathcal{F}$ such that $x^{*}$ is optimal for the composite integer programming problem (20).

Proof. First assume that $x^{*}$ is an efficient solution of (5). By Theorem 4 there exists an index $i \in\{1, \ldots, k\}$ and lower bounds $e_{j} \in \mathbb{R}, j \in J_{i}$ such that $x^{*}$ is 
an optimal solution of (17). Let $F^{*}$ be the optimal solution of the corresponding dual problem (18). Then Theorem 9 implies that $x^{*}$ is optimal for (20).

Secondly, let $x^{*}$ be optimal for (20), and suppose that $x^{*}$ is not efficient for (5). Then there exists $\hat{x} \in \mathbb{R}^{n}$ such that $C \hat{x} \geq C x^{*}$. Let $i \in\{1, \ldots, k\}$ be an index for which $c^{i} \hat{x}>c^{i} x^{*}$. Then $C_{J_{i}} \hat{x} \geqq C_{J_{i}} x^{*}$, and since $F^{*}$ is nondecreasing it follows that $F^{*}\left(-C_{J_{i}} \hat{x}, b\right) \leq F^{*}\left(-C_{J_{i}} x^{*}, b\right)$. Hence

$$
c^{i} \hat{x}-F^{*}\left(-C_{J_{i}} \hat{x}, b\right)>c^{i} x^{*}-F^{*}\left(-C_{J_{i}} x^{*}, b\right),
$$

contradicting the optimality of $x^{*}$.

Once an efficient solution $x^{*}$ of (5) and a corresponding optimal dual function $F^{*} \in \mathcal{F}$ of (18) satisfying $c^{i} x^{*}=F^{*}\left(-C_{J_{i}} x^{*}, b\right)$ have been determined, Corollary 3 implies that $c^{i} x \leq F^{*}\left(-C_{J_{i}} x, b\right)$ for all $x \in\left\{x \in \mathbb{R}^{n}: A x \leqq b, x \geqq\right.$ 0 and integer $\}$. In the context of the MOIP (5) this implies that the level curve of the objective function of the corresponding composite integer programming problem (20) at level zero yields an upper bound on the set of nondominated solutions of (5) in the objective space.

Corollary 4. Let $x^{*}$ and $F^{*}$ be optimal for (17) and (18), respectively. Then the set $L_{F^{*}}(0):=\left\{z \in \mathbb{R}^{k}: z_{j}=c^{j} x \forall j \in J_{i}, z_{i}=F^{*}\left(-C_{J_{i}} x, b\right), A x \leqq b, x \geqq 0\right\}$ is an upper bound on the set of nondominated solutions $N$ of (5), i.e., $\forall z^{F} \in L_{F^{*}}(0)$ $\nexists z^{N} \in N$ s.t. $z^{N} \geq z^{F}$.

\section{Generation of Dual Optimal Functions and Composite Functions}

Dual optimal functions for single objective integer programming problems (14) can be generated by various different methods most of which are inspired by a solution method for the primal problem like, for example, cutting plane methods or branch and bound. In this way, the dual optimal functions are obtained as a byproduct of a solution of the original integer program and without additional computational effort.

\subsection{Generation of Dual Optimal Functions Using Cutting Planes}

We will use the Gomory fractional cutting plane algorithm (Gomory, 1963) as a representative example for a cutting plane method for the exact solution of integer programming problems. The following brief description is based on Wolsey (1981). 
Consider the integer program (14) where we, as before, assume that the problem is bounded and that all data is integer. Then in iteration $r$ of a cutting plane algorithm, $r \geq 0$, a linear programming problem $\left(P_{r}\right)$ given by

$$
\begin{array}{cl}
\max & c^{T} x \\
\text { s.t. } & \sum_{j=1}^{n} a_{i j} x_{j} \leq b_{i}, \quad i=1, \ldots, m+r \\
x \geqq 0 &
\end{array}
$$

is solved, where the first $m$ constraints are defined by the original constraint matrix $A$ and right-hand-side vector $b$ and the remaining $r$ constraints have been added during previous iterations. Note that in iteration $0,\left(P_{0}\right)$ is identical to the linear programming relaxation of (14).

If $\left(P_{r}\right)$ is infeasible or if the optimal solution is integer the algorithm terminates and the original problem (14) is solved. Otherwise, that is, if the optimal solution $x^{r}$ of $\left(P_{r}\right)$ has fractional components, there exists a constraint vector $a^{m+r+1}=\left(a_{m+r+1,1}, \ldots, a_{m+r+1, n}\right) \in \mathbb{R}^{n}$ and a right-hand side value $b_{m+r+1} \in \mathbb{R}$ such that $a^{m+r+1} x=b_{m+r+1}$ is a separating hyperplane between the set of integer feasible solutions of $\left(P_{r}\right)$ and the optimal solution $x^{r}$ of $\left(P_{r}\right)$. In particular, $a^{m+r+1}$ and $b_{m+r+1}$ can be found as

$$
\begin{aligned}
& a_{m+r+1, j}=G^{r+1}\left(a_{\bullet j}\right), \quad \text { where } \quad G^{r+1}(d):=\left\lfloor\sum_{i=1}^{m} \lambda_{i}^{r} d_{i}+\sum_{i=1}^{r} \lambda_{m+i}^{r} G^{i}(d)\right\rfloor, \\
& b_{m+r+1}=G^{r+1}(b), \quad \lambda^{r}=\left(\lambda_{1}^{r}, \ldots, \lambda_{m+r}^{r}\right) \geqq 0,
\end{aligned}
$$

see Chvatal (1973). (In this formulation, $\lambda^{r}$ is obtained as the fractional part of the vector of coefficients of the slack variables of the Gomory cut in its original form.)

We can conclude that $a^{m+r+1} x \leq b_{m+r+1}$ for all $x \in\left\{x \in \mathbb{R}^{n}: \sum_{j=1}^{n} a_{i j} x_{j} \leq\right.$ $b_{i}, i=1, \ldots, m+r, x$ integer $\}$, and $a^{m+r+1} x^{r}>b_{m+r+1}$. Hence the constraint $a^{m+r+1} x \leq b_{m+r+1}$ can be added as the $(m+r+1)^{\text {st }}$ constraint to $\left(P_{r}\right)$, that is, as a cutting plane that cuts off the fractional solution $x^{r}$ but no feasible solution of the original problem. The algorithm proceeds with the next iteration, increasing the iteration counter to $r+1$.

Based on this algorithm, dual optimal functions can be constructed as described in Wolsey (1981), utilizing the dual feasible solutions of the linear programming problems $\left(P_{r}\right)$ :

Lemma 1. Let $u^{r} \in \mathbb{R}^{m+r}, u^{r} \geqq 0$ be a dual feasible solution of $\left(P_{r}\right)$. Then the functions

$$
F^{r}(d):=\sum_{i=1}^{m} u_{i}^{r} d_{i}+\sum_{i=1}^{r} u_{m+i}^{r} G^{i}(d)
$$

are superadditive dual feasible functions for (14). Moreover, if $x^{r}$ is optimal for $\left(P_{r}\right)$ and $u^{r}$ is dual optimal for $\left(P_{r}\right)$, then $c^{T} x^{r}=F^{r}(b)$. 
Proof (see Wolsey, 1981). The second part of the lemma follows immediately from linear programming duality, c.f. Section 3.2. For the first part, observe that $F^{r}\left(a_{\bullet j}\right) \geq c_{j}$ for all $j=1, \ldots, n$ since $u^{r}$ is dual feasible for $\left(P_{r}\right)$. Moreover, $F^{r} \in \mathcal{G}$ (c.f. Section 3.3) since $u^{r} \geqq 0, \lambda^{i} \geqq 0$ for all $i=1, \ldots, r-1$ and $G^{i}\left(d_{1}\right)+G^{i}\left(d_{2}\right) \leq G^{i}\left(d_{1}+d_{2}\right)$ for all $d_{1}, d_{2} \in \mathbb{R}^{m}, i=1, \ldots, r($ as $\lfloor\alpha\rfloor+\lfloor\beta\rfloor \leq$ $\lfloor\alpha+\beta\rfloor$ for all $\alpha, \beta \in \mathbb{R})$.

Under the assumption that the Gomory cutting plane algorithm terminates after a finite number of iterations $r$ (which is, for example, guaranteed if the feasible set of $\left(P_{0}\right)$ is bounded), the above lemma implies that a dual optimal function of (14) can be constructed iteratively using functions of the form (22):

Theorem 11. Suppose that for a given problem (14) the Gomory cutting plane algorithm terminates after a finite number of $r$ iterations. If (14) has a finite optimal solution, then there exists an optimal feasible solution $x^{r}$ of (14) and a dual optimal function $F^{r}$ of (16) of the form (22) such that $c^{T} x^{r}=F^{r}(b)$.

Moreover, if (14) is infeasible, then there exists a dual function $F^{r}$ of the form (22) satisfying $F^{r}\left(a_{\bullet j}\right) \geq 0, j=1, \ldots, n$ and $F^{r}(b)<0$. In this case the dual (16) of (14) is unbounded.

Example 1. Consider the integer programming problem

$$
\begin{aligned}
& \max x_{1}+x_{2} \\
& \text { s.t. }-x_{1} \leq-3 \\
& 5 x_{1}+2 x_{2} \leq 22 \\
& x_{2} \leq 6 \\
& x_{1}, x_{2} \geq 0 \text { and integer. }
\end{aligned}
$$

The optimal solution of the inital LP relaxtion $\left(P_{0}\right)$ is obtained as $x^{0}=(3 ; 3.5)^{T}$ with objective value $z_{0}=6.5$. Since the solution has fractional components the first Gomory cut is determined, defining the additional constraint $2 x_{1}+x_{2} \leq 9$. Problem $\left(P_{1}\right)$ of iteration 1 is then given by

$$
\begin{array}{lrl}
\max & x_{1}+x_{2} & \\
\text { s.t. } & -x_{1} & \\
& 5 x_{1}+2 x_{2} & \leq 22 \\
& \\
2 x_{1}+x_{2} & \leq 6 \\
& & \leq 9 \\
& x_{1}, x_{2} \leq 0
\end{array}
$$

with optimal solution $x^{1}=(3 ; 3)^{T}$ and objective value $z_{1}=6$. As this solution is feasible for (23) the algorithm terminates. The corresponding dual optimal solution is determined as $u^{1}=(1 ; 0 ; 0 ; 1)$, and with $\lambda^{1}=\left(\frac{1}{2} ; \frac{1}{2} ; 0\right)$ we obtain a dual optimal function according to $(22)$ as

$$
F_{C P}(d):=F^{r}(d)=d_{1}+G^{1}(d)=d_{1}+\left\lfloor\frac{1}{2}\left(d_{1}+d_{2}\right)\right\rfloor .
$$




\subsection{Generation of Dual Optimal Functions Using Branch and Bound}

While in the previous section superadditive dual optimal functions were generated, the application of a branch and bound algorithm for the solution of integer programming problems (14) will in general only yield nondecreasing dual optimal functions in the set $\mathcal{F}$, c.f. Section 3.3.

The basic idea of a branch and bound algorithm is to replace the original problem $(14)$ by a finite series of subproblems $\left(P_{t}\right)$ of the form

$$
\begin{array}{ll}
\max & c^{T} x \\
\text { s.t. } & A x \leqq b \\
& x=X_{t},
\end{array}
$$

$t=1, \ldots, r$, such that $\left\{x \in \mathbb{R}^{n}: x \geqq 0\right.$ and integer $\} \subseteq \bigcup_{t=1}^{r} X_{t}$. We will assume in the following that the sets $X_{t}$ are given as $X_{t}=\left\{x \in \mathbb{R}^{n}: g_{j}^{t} \leq x_{j} \leq h_{j}^{t}, j=\right.$ $1, \ldots, n, x \geqq 0\}$ where $g_{j}^{t} \geq 0$ and $h_{j}^{t}$ are integer lower and upper bounds on the variables $x_{j}, j=1, \ldots, n$. This assumption is satisfied by a majority of branch and bound algorithms, and in particular by linear programming based approaches that rely on the solution of a series of linear programming problems $\left(P_{t}\right)$.

A branch and bound algorithm terminates if all of the subproblems are shown to be infeasible, or if an integer solution $x^{t^{*}}$ is found that is optimal for some subproblem $\left(P_{t^{*}}\right)$ and whose objective value $z_{t^{*}}=c^{T} x^{t^{*}}$ dominates all of the remaining subproblems, that is, $z_{t^{*}} \geq z_{t}$ for all $t \neq t^{*}$ where $z_{t}$ is the optimal objective value of subproblem $\left(P_{t}\right)$. Otherwise, one or several subproblems are further devided to obtain better solutions and / or bounds.

Lemma 2. If $F_{t} \in \mathcal{F}$ are dual feasible functions for $\left(P_{t}\right)$ in the sense that $F_{t}(A x) \geq c^{T} x \forall x \in X_{t}, t=1, \ldots, r$, then

$$
F(d):=\max _{t=1, \ldots, r} F_{t}(d)
$$

is a dual feasible function for (14).

Proof (see Wolsey, 1981). Let $x \geqq 0$ and integer. Then $x \in X_{t}$ for some $t \in\{1, \ldots, r\}$ which implies that $F(A x) \geq F_{t}(A x) \geq c^{T} x$. Moreover, $F$ is nondecreasing since $F_{t}, t=1, \ldots, n$, are nondecreasing, and thus $F \in \mathcal{F}$.

Theorem 12. Let (14) have a finite optimum solution. If a linear programming based branch and bound algorithm terminates with a finite series of subproblems $\left(P_{t}\right), t=1, \ldots, r$, then there exists a dual optimal function $F \in \mathcal{F}$ of the form

$$
F(d):=\max _{t=1, \ldots, r}\left(\pi^{t} d+\alpha^{t}\right), \quad \alpha^{t} \in \mathbb{R}, \pi^{t} \in \mathbb{R}^{m}, \pi^{t} \geqq 0
$$


Proof (see Wolsey, 1981). Let $z^{*}$ be the optimum objective value of (14) and let $t \in\{1, \ldots, r\}$ be an arbitrary subproblem $\left(P_{t}\right)$ at the termination of the algorithm.

If the linear programming problem $\left(P_{t}\right)$ has a finite optimum objective value $z_{t}$ the corresponding LP dual is feasible. Thus the dual optimal solution $\left(\pi^{t}, \underline{\pi}^{t}, \bar{\pi}^{t}\right) \geqq$ 0 satisfies $\pi^{t} a_{\bullet j}-\sum_{j=1}^{n} \underline{\pi}_{j}^{t}+\sum_{j=1}^{n} \bar{\pi}_{j}^{t} \geq c_{j}, j=1, \ldots, n$, and the function $F_{t}(d)=\pi^{t} d+\alpha^{t}$ with $\alpha^{t}:=-\underline{\pi}^{t} g^{t}+\bar{\pi}^{t} h^{t}$ satisfies $F_{t}(A x)=\pi^{t} A x+\alpha^{t}=$ $\pi^{t} A x-\underline{\pi}^{t} g^{t}+\bar{\pi}^{t} h^{t} \geq \pi^{t} A x-\underline{\pi}^{t} x+\bar{\pi}^{t} x \geq c^{T} x \forall x \in X_{t}$. Hence $F_{t}$ is dual feasible for $\left(P_{t}\right)$ in the sense of Lemma 2. Moreover, by linear programming duality, $F_{t}(b)=\pi^{t} b-\pi^{t} g^{t}+\bar{\pi}^{t} h^{t}=z_{t}$ where $z_{t} \leq z^{*}$ at the termination of the algorithm.

If, on the other hand, the linear programming problem $\left(P_{t}\right)$ is infeasible, there exists a dual ray $\left(\omega^{t}, \underline{\omega}^{t}, \bar{\omega}^{t}\right) \geqq 0$ such that $\omega^{t} a_{\bullet j}-\sum_{j=1}^{n} \underline{\omega}_{j}^{t}+\sum_{j=1}^{n} \bar{\omega}_{j}^{t} \geq 0$ and $\omega^{t} b-\underline{\omega}^{t} g^{t}+\bar{\omega}^{t} h^{t}<0$. This dual ray can be combined with any dual feasible solution $\left(\pi^{k}, \underline{\pi}^{k}, \bar{\pi}^{k}\right) \geqq 0$ of the LP dual of $\left(P_{t}\right)$ (which generally is available from a predecessor node $\left(P_{k}\right)$ in the branch and bound tree) to define a vector $\left(\pi^{t}, \underline{\pi}^{t}, \bar{\pi}^{t}\right):=\left(\pi^{k}, \underline{\pi}^{k}, \bar{\pi}^{k}\right)+\lambda\left(\omega^{t}, \underline{\omega}^{t}, \bar{\omega}^{t}\right)$ with a positive scalar $\lambda \in \mathbb{R}$. A dual feasible function of $\left(P_{t}\right)$ in the sense of Lemma 2 is then obtained as $F(d)=$ $\pi^{t} d+\alpha^{t}$ with $\alpha^{t}:=-\underline{\pi}^{t} g^{t}+\bar{\pi}^{t} h^{t}$. Note that $\lim _{\lambda \rightarrow \infty} F_{t}(b)=-\infty$ and hence $\lambda$ can always be chosen such that $F_{t}(b)<z^{*}$.

We can conclude that $F(d)=\max _{t=1, \ldots, r} F_{t}(d)$ is a dual feasible function for (14) according to Lemma 2. Moreover, for an optimal solution $x^{*}$ of (14) there exists a subproblem $\left(P_{t^{*}}\right)$ such that $x^{*}$ is optimal for $\left(P_{t^{*}}\right)$ and $z^{*}=c^{T} x^{*}=F_{t^{*}}(b)$. Since $F_{t}(b) \leq z^{*}$ for all $t=1, \ldots, r$ it follows that $F(b)=\max _{t=1, \ldots, r} F_{t}(b)=$ $F_{t^{*}}(b)=z^{*}$.

Example 2. Consider again the integer programming problem (23) introduced in Example 1. The branch and bound tree generated by the algorithm is shown in Figure 1.

At the termination of the algorithm, the partition consists of the subproblems $\left(P_{3}\right),\left(P_{4}\right)$ and $\left(P_{5}\right)$. The corresponding dual functions in the sense of Lemma 2 are given by

$$
\begin{aligned}
F_{3}(d) & =[(1.5 ; 0.5 ; 0)+\lambda(5 ; 1 ; 0)] d-[0+\lambda \cdot 2] \cdot 4 \\
& =(6.5 ; 1.5 ; 0) d-8=6.5 d_{1}+1.5 d_{2}-8 \\
F_{4}(d) & =(0 ; 0 ; 0) d+(1 ; 1)(3 ; 3)^{T}=6 \\
F_{5}(d) & =(0 ; 0.5 ; 0) d+0 \cdot 3-1.5 \cdot 4=0.5 d_{2}-6 .
\end{aligned}
$$

Hence an optimal dual function of (23) is obtained as

$$
F_{B B}(d)=\max _{t=3,4,5} F_{t}(d)=\max \left\{6.5 d_{1}+1.5 d_{2}-8 ; 6 ; 0.5 d_{2}-6\right\} .
$$




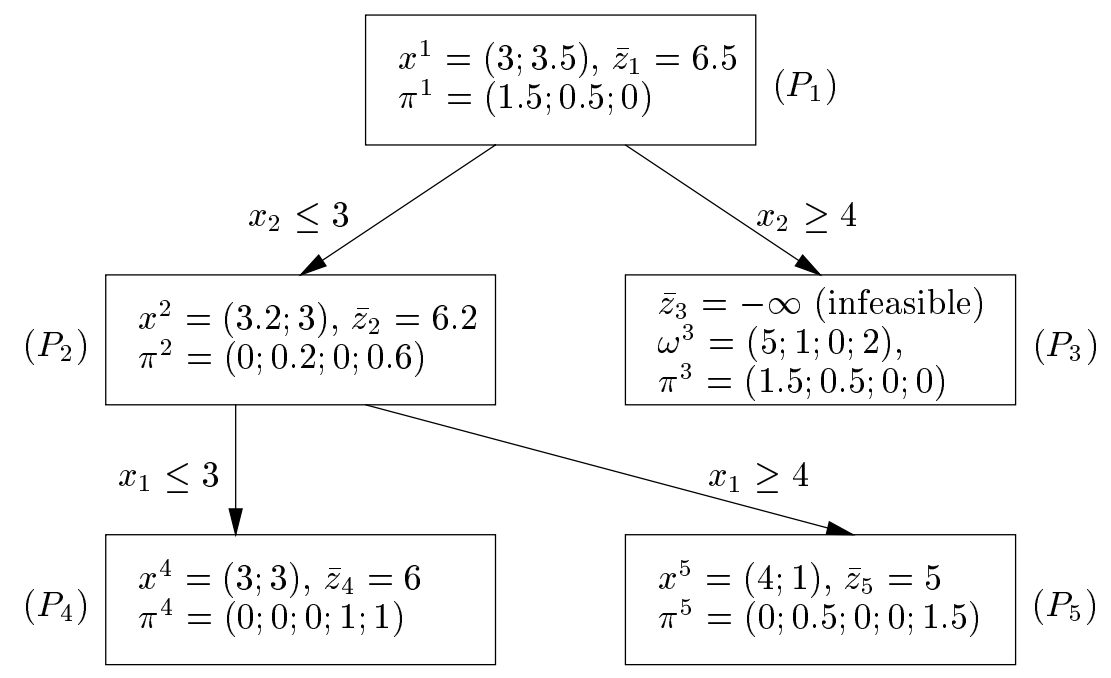

Figure 1: Branch and bound tree for the example problem (23).

\subsection{Composite Functions Based on Cutting Planes and Branch and Bound}

Both methods discussed in the previous sections can be used to generate dual optimal functions and hence composite functions for MOIPs as discussed in Section 4. This approach will be demonstrated on the following example, building upon Examples 1 and 2.

Example 3. Consider the MOIP

$$
\begin{aligned}
& \max x_{1}=z_{1} \\
& \max x_{1}+x_{2}=z_{2} \\
& \text { s.t. } 5 x_{1}+2 x_{2} \leq 22 \\
& x_{2} \leq 6 \\
& x_{1}, x_{2} \geq 0 \text { and integer. }
\end{aligned}
$$

The $e$-constraint LP obtained by selecting the second objective for individual maximization (i.e., $i=2$ ) and fixing the lower bound on the first objective to 3 (i.e., $e_{1}=3$ ) is identical to problem (23) introduced in Example 1. Figure 2 shows the set of feasible solutions of (25) in the objective space together with the dual optimal functions of (23) as found in Examples 1 and 2. Here the value of $e_{1}$ is considered as a variable which implies that for values of $e_{1}$ different from $e_{1}=3$ the dual functions yield upper bounds on the values of the second objective $z_{2}$.

Depending on the method used for constructing the optimal dual functions, the following composite integer programming problems are obtained: 

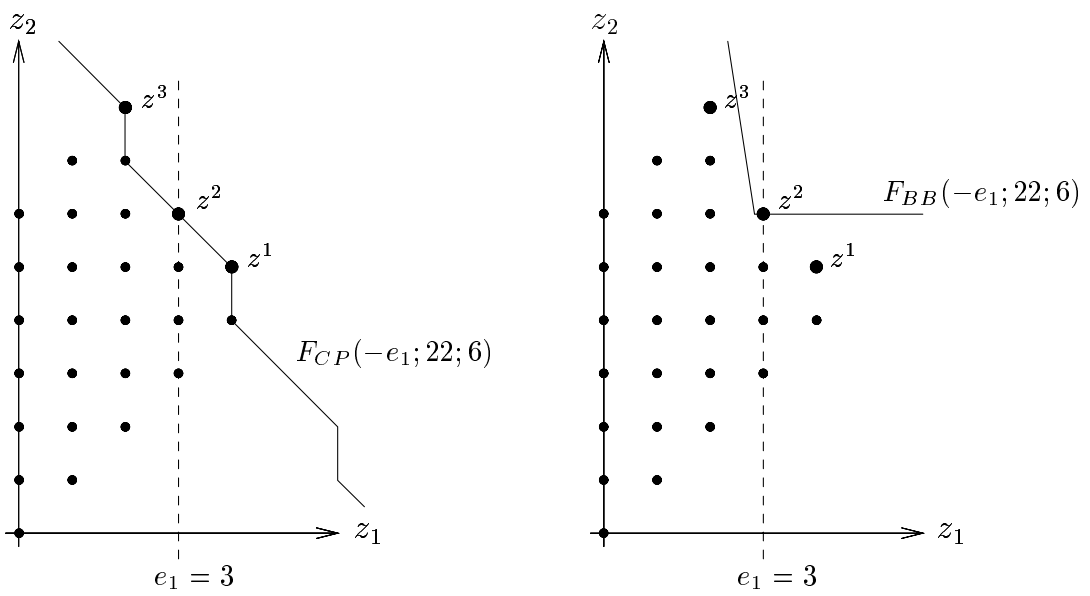

Figure 2: Dual functions generated based on (a) cutting planes and (b) branch and bound.

- For the cutting planes method:

$$
\begin{array}{ll}
\max & x_{1}+x_{2}-F_{C P}\left(-x_{1} ; 22 ; 6\right)=2 x_{1}+x_{2}-\left\lfloor-0.5 x_{1}+11\right\rfloor \\
\text { s.t. } & 5 x_{1}+2 x_{2} \leq 22 \\
x_{2} & \leq 6 \\
x_{1}, x_{2} & \geq 0 \text { and integer. }
\end{array}
$$

- For the branch and bound algorithm:

$$
\begin{array}{ll}
\max & x_{1}+x_{2}-F_{B B}\left(-x_{1} ; 22 ; 6\right)=x_{1}+x_{2}-\max \left\{-6.5 x_{1}+25 ; 6 ; 5\right\} \\
\text { s.t. } & 5 x_{1}+2 x_{2} \leq 22 \\
& x_{2} \leq 6 \\
x_{1}, x_{2} & \geq 0 \text { and integer. }
\end{array}
$$

Note that the representations of the dual functions shown in Figure 2 correspond to the level curves of the objective functions of the respective composite integer programming problems at level zero. These level curves constitute two distinct outer approximations of the set of nondominated solutions of (25), c.f. Corollary 4.

\section{Conclusions}

Based on dual information obtained from appropriately formulated single objective linear and integer programming problems, we have developed an integrated utility theory for multiple objective linear programming (MOLP) and multiple objective integer programming (MOIP). The suggested composite function approach leads to a unifying theory that highlights the close relationship of the two models as well as their intrinsic differences. 
Composite functions can be used to evaluate and visualize utility information yielding a specific nondominated solution. Their determination can be integrated with applications of classical cutting plane methods or branch and bound algorithms for the solution of $e$-constraint versions of (MOIP). This naturally suggests a combination of composite function determinations within an interactive procedure that repeatedly formulates and solves e-constraint problems, see Bard (1986). In particular, the upper bounds on the nondominated set obtained from level curves of composite functions provide valuable information which can be used to aid the decision maker with the adaptation of aspiration levels and / or the selection of a most relevant optimization objective, also in the context of aspiration-based methods as suggested in Nakayama (1995) and Miettinen and Mäkelä (2000).

In applications where an exact determination of composite functions using, for example, branch and bound is impractible due to the size of the branch and bound tree, approximate composite functions (and weaker upper bounds) can be obtained based on premature termination of a branch and bound (or cutting plane) algorithm. Already after a few branching steps the resulting approximate composite functions are likely to yield improved utility information as compared to simple weighted sums scalarizations obtained from the LP relaxation of (MOIP).

\section{References}

Alves, M. J. and Clímaco, J. (1999). Using cutting planes in an interactive reference point approach for multiobjective integer linear programming. European Journal of Operational Research, 117, 565-577.

Alves, M. J. and Clímaco, J. (2000). An interactive reference point approach for multiobjective mixed-integer programming using branch-and-bound. European Journal of Operational Research, 124, 478-494.

Bard, J. F. (1986). A multiobjective methodology for selecting subsystem automation options. Management Science, 32, 1628-1641.

Chankong, V. and Haimes, Y. Y. (1983a). Multiobjective Decision Making: Theory and Methodology. Elsevier Science Publishing, New York.

Chankong, V. and Haimes, Y. Y. (1983b). Optimization-based methods for multiobjective decision-making: An overview. Large Scale Systems, 5, 1-33.

Chvatal, V. (1973). Edmonds polytopes and a hierarchy of combinatorial problems. Discrete Mathematics, 4, 305-337. 
Clímaco, J., Ferreira, C., and Captivo, M. (1997). Multicriteria integer programming: An overview of the different algorithmic approaches. In J. Clímaco, editor, Multicriteria Analysis, pages 248-258. Springer, Berlin, Heidelberg, New York.

Cohon, J. L. (1978). Multiobjective Programming and Planning. Academic Press, New York.

Gomory, R. E. (1963). An algorithm for integer solutions to linear programs. In R. L. Graves and P. Wolfe, editors, Recent Advances in Mathematical Programming, pages 269-302. McGraw-Hill, New York.

Haimes, Y., Lasdon, L., and Wismer, D. (1971). On a bicriterion formulation of the problems of integrated system identification and system optimization. IEEE Transactions on Systems, Man, and Cybernetics, 1, 296-297.

Jahn, J. (1983). Duality in vector optimization. Mathematical Programming, 25, $343-353$.

Klabjan, D. (2002). A new subadditive approach to integer programming: Theory and algorithms. In Proceedings of the Ninth Conference on Integer Programming and Combinatorial Optimization, Cambridge, MA, pages 384-400.

Llewellyn, D. and Ryan, J. (1993). A primal dual integer programming algorithm. Discrete Applied Mathematics, 45, 261-275.

Miettinen, K. and Mäkelä, M. M. (2000). Interactive multiobjective optimization system WWW-NIMBUS on the internet. Computers and Operations Research, 27, 709-723.

Nakayama, H. (1995). Aspiration level approach to interactive multi-objective programming and its applications. In P. Pardalos, Y. Siskos, and C. Zopounidis, editors, Advances in Multicriteria Analysis, pages 147-174. Kluwer, Dordrecht.

Nemhauser, G. L. and Wolsey, L. A. (1986). Integer and Combinatorial Optimization. Wiley \& Sons, New York.

Schrijver, A. (1986). Theory of Integer and Linear Programming. Wiley \& Sons, New York.

Steuer, R. E. (1986). Multiple Criteria Optimization: Theory, Computation, and Application. Wiley \& Sons, New York.

Tind, J. and Wolsey, L. A. (1981). An elementary survey of general duality theory in mathematical programming. Mathematical Programming, 21, 241-261. 
Visée, M., Teghem, J., Pirlot, M., and Ulungu, E. L. (1998). Two-phases method and branch and bound procedures to solve the bi-objective knapsack problem. Journal of Global Optimization, 12, 139-155.

Wolsey, L. A. (1981). Integer programming duality: Price functions and sensitivity analysis. Mathematical Programming, 20, 173-195.

Wolsey, L. A. (1998). Integer Programming. Wiley \& Sons, New York. 